\title{
Endovascular Repair of Peripheral Arterial Aneurysms
}

\author{
Nabil A. MD, MSc; ${ }^{1}$ Zaki M., MD, MSc, MRCS; ${ }^{1}$ Kerdawi S. MD, MSc ${ }^{2}$ \\ ${ }^{1}$ Department of Vascular Surgery, Faculty of Meidcine, Ain Shams University, Egypt \\ ${ }^{2}$ Wadi El Nil Hospital, Cairo, Egypt
}

\begin{abstract}
Aim: To assess the feasibility of using endovascular techniques as a safe and effective method in treating high risk patients with peripheral aneurysms

Patient and methods: Our series includes 15 peripheral aneurysms treated in our Unit with endovascular technique in the period between January 2014 and February 2015. Of these 11 were men (73.3\%) and 4 women (26.6\%) with a mean age of 67.2 years (range $45-71$ years). The localization of the aneurysmal lesions was as follows: 6 at femoral artery level (4 pseudo-aneurysm and 2 true), 4 iliac artery(CIA, internal iliac) (true), 2 popliteal artery (true), 2 anterior tibial artery (pseudo-aneurysm) and one subclavian (true). The average length of the lesion was found to be $47.3 \mathrm{~mm}$ (range $33-67 \mathrm{~mm}$ ). We used either coils or covered stent to treat such aneurysms.The follow-up was done by serial outpatient clinical evaluation and Doppler-US and CT angiography every 6 months.
\end{abstract}

Results: The clinical success was immediate in 14 patients (93.3\%): In 1 case femoral artery aneurysm has not been completely excluded. The follow-up was done by serial outpatient clinical evaluation and Doppler-US and CT angiography every 6 months to check the correct positioning of the stent and good exclusion of the aneurysm after coiling for a minimum period of 12 months.

Conclusion: The reconstruction of endovascular aneurysm of peripheral arteries has been proposed in recent decades as an alternative to conventional surgical treatment in patients at high surgical risk.

Key words: Peripheral arterial aneurysm, endovascular repair, coiling, coverd stent.

\section{Introduction}

Arterial aneurysm is known as an enlargement of an artery more than one and half times its normal diameter. True aneurysms characterized by intact arterial wall which has all three layers (intima, media and adventitia), and are usually atherosclerotic or degenerative in nature. In contrast, false or pseudoaneurysms lack a complete arterial wall, and are usually caused by trauma, iatrogenic injury or infection. ${ }^{1}$

Peripheral aneurysms may affect arterial tree other than aorta as visceral arteries, renal artery, supra-aortic trunk and upper or lower limb arterial aneurysms. Lower extremity arterial aneurysms are the most frequent peripheral aneurysms. In practical point of view, most of femoral or tibial arteries aneurysms are false aneurysms and iatrogenic in nature, while those of the popliteal artery are true atherosclerotic and degenerative aneurysms. ${ }^{2}$

Iatrogenic femoral false aneurysms may occur at the anastomotic site of previous operation (arterial repair or bypass) or as a side effect of common femoral artery punctures in cardiac PCI. ${ }^{1}$ Less frequently, false aneurysms of distal arteries, such as superficial, deep femoral, popliteal or tibial arteries, caused by a traumatic (usually penetrating) surgical or orthopedic procedure. False aneurysms mostly presented by pulsating hematoma or pulsating painful swelling that may rupture or lead to distal embolization, and also progressive enlargement may lead to overlying skin necrosis and sloughing. ${ }^{2}$

Femoral puncture false aneurysms managed conservatively when it is small less than $2 \mathrm{~cm}$ by stoppage of anticoagulation if not possible, ultrasound guided compression or thrombin injection. Larger ones from a femoral puncture and anastomotic or traumatic false aneurysms should be repaired either by open surgical repair (debridement and direct repair or bypass) or by placing of covered stent via contralateral approach.

The most common true lower extremity aneurysms are popliteal artery aneurysms (70\%), with an incidence approximately of $0.4 \%$ in hospitalized patients. ${ }^{3}$ Femoral artery aneurysms are commonly associated with popliteal aneurysms and are the second most common peripheral aneurysms.

Also, there is a strong association between true femoropopliteal aneurysms and with the other limb, as well as with the aorto iliac true aneurysms: In patients with a femoropopliteal aneurysm, the prevalence of an aortic aneurysm is about $50 \% .{ }^{4}$ 
Moreover, both femoral and popliteal artery aneurysms are found in association in 50\% of patients bilaterally. ${ }^{5}$ A femoropopliteal aneurysm is found in $10 \%$ of patients developed abdominal aortic aneurysm. Risk factors of femoropopliteal true aneurysms are similar of those for aortic aneurysm like family history, male gender, smoking, hypertension and hyperlipidemia.

Clinical presentation of true common femoral artery aneurysms differ from painless pulsating groin swelling to painful, tender pulsating mass due to femoral nerve neuralgia and limb oedema.

Embolization from laminated thrombus may lead to limb ischemia (claudication or critical limb ischemia), also acute thrombosis may occur in $15 \%$ of cases, while rupture is uncommon. ${ }^{4}$

Popliteal aneurysms is mostly asymptomatic, pulsating painless swelling in the popliteal fossa and may develop complications of chronic or acute ischemia, whereas rupture is uncommon. Ischemic symptoms of popliteal aneurysm are due to thrombosis of the aneurysm or distal showers in the tibial arteries, the incidence of developing symptoms is 34\% at 3 years in asymptomatic patients having intact distal pulse increased to $86 \%$ in patients with no pedal pulse. ${ }^{6}$

Acute limb ischemia is the most serious complication of popliteal aneurysms, and carry high risk of limb loss. Treatment is designed according to clinical judgment, which ranges from intravenous heparin, catheter directed thrombolysis, emergency surgical bypass or primary amputation. ${ }^{7}$ Because the results of surgical treatment are best in asymptomatic patients, and become worse and carry high risk of amputation in patients with chronic or acute limb ischemia, detecting popliteal aneurysms in the asymptomatic stage is mandatory.

The indications for elective treatment are diameter more than $2.5 \mathrm{~cm}$ unless the aneurysm develop mural thrombus, embolization, or local mass effect. The primary aim of treatment is to exclude the aneurysm from the circulation, and an open approach with surgical bypass plus ligation of the aneurysm, or with an interposition graft, is nowadays still the treatment of choice.

With the emerge of endovascular techniques, endovascular popliteal artery repair (EPAR) has become a promising and widespread treatment option. As with endovascular aortic aneurysm repair, EPAR is a technique that uses a covered stent to exclude the aneurysm and therefore is equally constrained, primarily by anatomy. ${ }^{8}$

\section{Patients and methods}

Our series includes 15 peripheral aneurysms, most of them in high risk individuals, treated at our Units in Ain Shams University Hospitals and Wadi El Nil Hospitals with endovascular technique in the period January 2015 - Feb 2016. Of these 11 were men $(73.3 \%)$ and 4 women $(26.6 \%)$ with a mean age of 67.2 years (range $45-71$ years). The localization of the aneurysmal lesions was as follows: 6 at femoral artery level (4 pseudoanurysm and 2 true), 4 iliac artery (true), 2 popliteal artery (true), 2 anterior tibia artery (pseudoanurysm) and one subclavian (true). The average length of the lesion is found to be $47.3 \mathrm{~mm}$ (range $33-67 \mathrm{~mm}$ ). For all patients we used the femoral access (ipsilateral in 4 cases, contralateral in the 6 cases and retrograde femoral in 2 cases).

After written informed consent, all patients were subjected to physical and instrumental examination. After local anesthesia with lidocaine $2 \%$ in site of access with or without conscious sedation, and antibiotic prophylaxis with intravenous single dose of cefazoline or ceftriaxone, the introductor $6-8 \mathrm{~F}$ was introduced through common femoral artery and was made to cannulate the district concerned. After iodinated contrast medium injection $50 \%$ diluted, a catheter was advanced and we proceeded to stenting. 12 patients $(80 \%)$ were treated by placement of covered stents(Viabahn or V12), $3(20 \%)$ with coilling of internal iliac artery (Figure 1) or ATA.

We infused 4000-6000 IU of low molecular weight heparin. Then, we verified the successful exclusion of the aneurysm. Removing the introducer we proceeded to perform hemostasis by manual or device closure. Patients were mobilized after 24 hours, and they were discharged after 72 hours. Planned follow-up was at 12 months.

\section{Results}

The clinical success was immediate in 14 patients (93.3\%); in 1 case femoral artery aneurysm was not completely excluded due to the high vessel tortuosity and the excessive length of the arterial segment affected by the lesion. We had no periprocedural complication. There was no procedure related or 30 day mortality; however, a single mortality $(3.7 \%)$ was recorded after 6 months of treatment from preexisting cardiac compromise (Table 1).

The follow-up was done by serial outpatient clinical evaluation and Doppler-US and CT angiography every 6 months to check the correct positioning of the stent and good exclusion of the aneurysm after coiling for a minimum period of 12 months.

During the follow-up were found one thrombotic episodes into the SFA stent one day after which 
was managed by thrombolysis, and one episode of device migration in the Popliteal aneurysm managed by another stent extension.

Our experience suggests that the endovascular exclusion of the aneurysmal lesions of the popliteal artery may require the application of one or more endoprosthesis whose total length exceeds about $3 \mathrm{~cm}$ of the length of aneurysm, both proximally and distally. In order to prevent the disconnection between the devices is preferable to realize an overlapping of at least $3 \mathrm{~cm}$ between the endoprosthesis, which may differ by one millimeter in diameter. The considerable flexion and repeated movements of the knee can cause kinking of the device, resulting in thrombosis, and cause breakage or migration of the stent.

This is an example of a left internal iliac aneurysm (Figure 1) in a 45 male patient, hepatic which was discovered accidentally during routine work up, surgical repair is an option but was risky due to the liver condition, so coiling was done via contralateral approach with complete exclusion of the 2 internal iliac aneurysms under local anesthesia.

Table 1

\begin{tabular}{|c|c|c|}
\hline & & $\begin{array}{c}\text { Number } \\
\%\end{array}$ \\
\hline \multirow{2}{*}{ Gender } & Male & $11(73.3 \%)$ \\
\hline & Female & $4(26.6 \%)$ \\
\hline \multirow{2}{*}{ DM } & Yes & $8(53.3 \%)$ \\
\hline & No & $7(46.7 \%)$ \\
\hline \multirow{2}{*}{ Hypertension } & Yes & $10(66.7 \%)$ \\
\hline & No & $5(33.3 \%)$ \\
\hline \multirow{2}{*}{ Chronic kidney disease } & Yes & $1(6.7 \%)$ \\
\hline & No & $14(93.3 \%)$ \\
\hline \multirow{2}{*}{ Ischemic heart disease } & Yes & $9(60 \%)$ \\
\hline & No & $6(30 \%)$ \\
\hline \multirow{2}{*}{ Chronic liver disease } & Yes & $2(13.3)$ \\
\hline & No & $13(86.6)$ \\
\hline \multirow{5}{*}{ Aneurysm location } & Femoral & 6 \\
\hline & Iliac & 4 \\
\hline & Popliteal & 2 \\
\hline & Anterior tibial & 2 \\
\hline & Subclavian & 1 \\
\hline \multirow{2}{*}{ Type of aneurysm } & True aneurysm & 9 \\
\hline & Pseudoaneurysm & 6 \\
\hline \multirow{3}{*}{ Access } & Ipsilateral antegrade & 4 \\
\hline & Contralateral retrograde & 6 \\
\hline & Ipsilateral Retrograde & 2 \\
\hline \multirow{2}{*}{ Management } & Coiling & $\begin{array}{c}3 \\
(20 \%)\end{array}$ \\
\hline & Covered stents & $\begin{array}{c}12 \\
(80 \%)\end{array}$ \\
\hline \multirow{3}{*}{ Immediate technical Success } & Yes & $\begin{array}{c}14 \\
(93.3 \%)\end{array}$ \\
\hline & No & $\begin{array}{c}1 \\
(6.7 \%)\end{array}$ \\
\hline & Mean & SD \\
\hline Age & 67.2 & 12.5 \\
\hline Lesion length & $47.3 \mathrm{~mm}$ & $21.7 \mathrm{~mm}$ \\
\hline
\end{tabular}




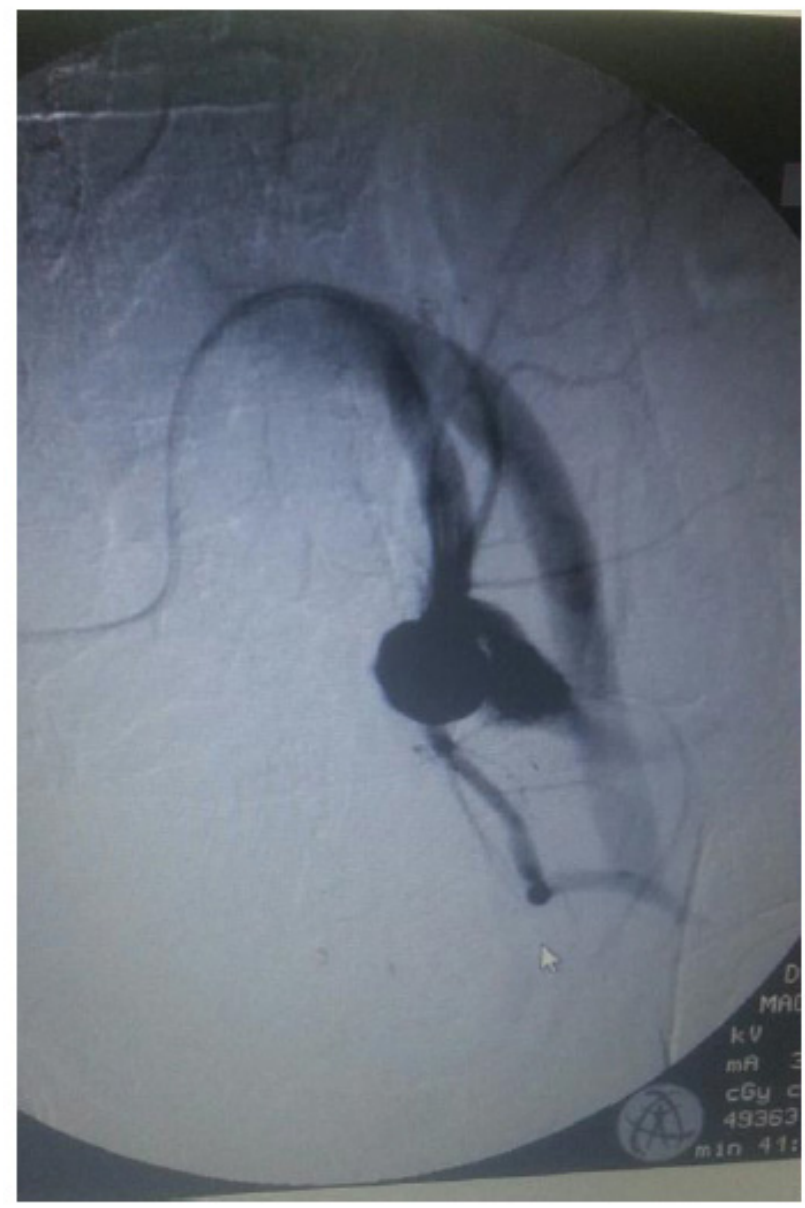

Fig 1: Internal iliac artery aneurysm

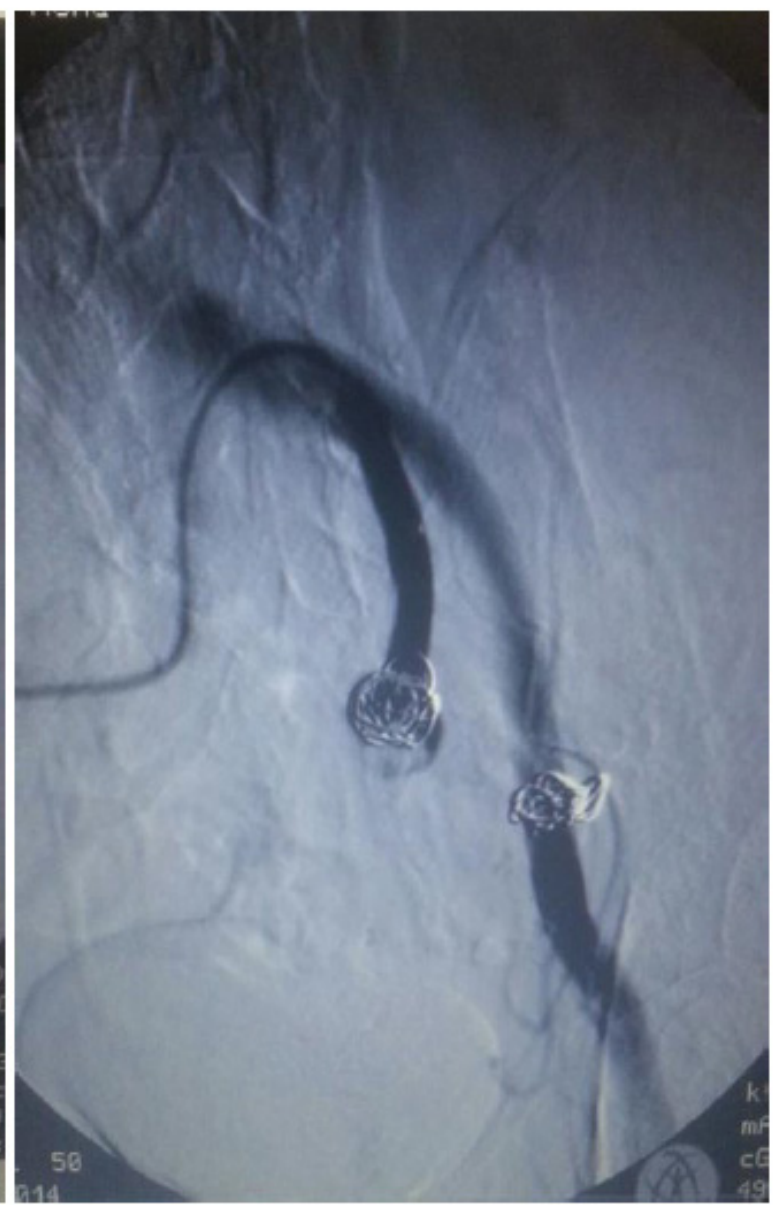

Post internal iliac artery coiling.

\section{Discussion}

Peripheral arterial aneurysms are a collection of conditions where the same pathology presents in multiple anatomic locations. They are often detected incidentally and the aetiology, natural history, clinical presentation and treatment of peripheral aneurysms may differ completely, depending on their site. Peripheral aneurysms have an underestimated morbidity and mortality risk, and elective treatment has been demonstrated to prevent complications such as rupture or embolisation. ${ }^{5}$ Although endovascular repair has become increasingly popular, conventional open surgical repair has continued to play a substantial role in the treatment of non-aortic aneurysms. Treatment must be tailored to the patient and anatomic location, and early counselling and assessment by a vascular specialist is recommended. ${ }^{8}$

Nowadays, it is increasingly common to use endovascular technique with use of endoprostheses for the treatment of such pathology. Being a minimally invasive technique associated with low peri-and post-operative morbidity and mortality, it

is most often indicated in patients at high risk for surgery.

\section{Conclusions}

The reconstruction of endovascular aneurysm of peripheral arteries has been proposed in recent decades as an alternative to conventional surgical treatment in patients at high surgical risk. It is considered minimally invasive and eliminates the need to resort to blood transfusion. Endovascular treatment in peripheral aneurysm is therefore an effective and safe, with a high ratio of success in the short and medium term.

\section{References}

1. Kresowik TF, Khoury MD, Miller BV, et al: A prospective study of the incidence and natural history of femoral vascular complications after percutaneous transluminal coronary angioplasty. J Vasc Surg. 1991; 13: 328-33.

2. Corriere MA, Guzman RJ: True and false aneurysms of the femoral artery. Semin Vasc Surg. 2005; 18: 216-23. 
3. Lawrence PF, Lorenzo-Rivero S, Lyon JL: The incidence of iliac, femoral, and popliteal artery aneurysms in hospitalized patients. $\boldsymbol{J}$ Vasc Surg. 1995; 22: 409-15.

4. Chambers CM, Curci JA: Treatment of nonaortic aneurysms in the endograft era: Aneurysms of the innominate and subclavian arteries. Semin Vasc Surg. 2005; 18: 184-90.

5. Dent $T L$, Lindenauer SM, Ernst CB, Fry WJ: Multiple arteriosclerotic arterial aneurysms. Arch Surg. 1972; 105: 338-44.
6. Dawson I, Sie RB, van Bockel JH: Atherosclerotic popliteal aneurysm. Br J Surg. 1997; 84: 293-9.

7. Kropman RH, Schrijver AM, Kelder JC, Moll FL, de Vries JP: Clinical outcome of acute leg ischaemia due to thrombosed popliteal artery aneurysm: Systematic review of 895 cases. Eur J Vasc Endovasc Surg. 2010; 39: 452-7.

8. Tielliu IF, Verhoeven EL, Zeebregts CJ, Prins TR, Span MM, van den Dungen JJ: Endovascular treatment of popliteal artery aneurysms: Results of a prospective cohort study. $\boldsymbol{J}$ Vasc Surg. 2005; 41: 561-7. 